\title{
Primeiro registro de um Mephitidae (Mammalia: Carnivora) naturalmente infectado por parasitos do gênero Physaloptera (Rudolphi, 1918) (Spirurida: Physalopteridae)
}

\author{
Gregório Corrêa Guimarães \\ Thales Augusto Barçante * \\ Pedro Soares Bezerra Júnior \\ Amanda do Nascimento Oliveira \\ Matheus Camargos de Britto Rosa \\ Gabriela Castro Lopes \\ Joziana Muniz de Paiva Barçante \\ Universidade Federal de Lavras (UFLA), \\ Campus Universitário Reitor José Roberto Soares Scolforo \\ Caixa Postal 3037, CEP 37200-000, Lavras - MG, Brasil \\ * Autor para correspondência \\ tbarcante@gmail.com
}

Submetido em 09/06/2014

Aceito para publicação em 05/02/2015

\section{Resumo}

Os animais silvestres podem ser considerados reservatórios de espécies de vários parasitos. A ocorrência de determinados agentes parasitários pode fornecer informações importantes sobre a ecologia e o comportamento do hospedeiro e suas relações tróficas. Dessa forma, este estudo teve por objetivo determinar a fauna parasitária de animais silvestres do sul de Minas Gerais no período de janeiro a dezembro de 2011. Foi realizado um estudo transversal com uma amostra de conveniência constituída por cadáveres de dois animais atropelados, que foram resgatados de rodovias e transportados para o Laboratório de Anatomia Animal da Universidade Federal de Lavras (UFLA). Os exemplares foram inspecionados para verificação da presença de ectoparasitos e, em seguida, dissecados para recuperação do conteúdo gastrintestinal e detecção de helmintos. Não foram identificados ectoparasitos nos dois animais, ambos da espécie Conepatus semistriatus (jaritataca), mas foi identificada a presença de helmintos do gênero Physaloptera no estômago de um espécime.

Palavras-chave: Animais silvestres; Nematoide; Parasitos; Physaloptera

\section{Abstract}

First report of a Mephitidae (Mammalia: Carnivora) naturally infected by parasites of the genus Physaloptera (Rudolphi, 1918) (Spirurida: Physalopteridae). Wild animals may be regarded as reservoirs of several parasite species. The occurrence of certain parasitic agents may provide significant information on host's ecology and behavior and its trophic relations. Thus, this study aimed to determine the parasitic fauna of 
wild animals from southern Minas Gerais within the period from January to December 2011. A cross-sectional study was conducted with a convenience sample consisting of the dead bodies of two run over animals, which were rescued from highways and transported to the Laboratory of Animal Anatomy of the Federal University of Lavras (UFLA). The specimens were inspected to verify the presence of ectoparasites and, then, dissected to resume gastrointestinal content and detect helminths. No ectoparasites were identified in the two animals, both belonging to the species Conepatus semistriatus (striped hog-nosed skunk), but the presence of helminths belonging to the genus Physaloptera was identified in the stomach of one specimen.

Key words: Nematode; Parasites; Physaloptera; Wild animals

\section{Introdução}

Os cangambás pertencem à Ordem Carnivora, família Mephitidae (DRAGOO; HONEYCUTT, 1997), que é composta pelos gêneros Conepatus, Mephitis, Mydaus e Spilogale. Uma reorganização dos táxons do gênero Conepatus, com base em análises morfológicas e moleculares, reduziu para três o número de espécies válidas, na América do Sul: C. semistriatus (Boddarert 1785) e C. chinga (Molina 1782) - ocorrendo no Brasil e C. humboldti (Gray 1837) na Argentina e Chile (DRAGOO et al., 2003). Apesar de ser de ocorrência bastante frequente, são poucos os dados sobre ecologia, comportamento, distribuição, delimitação taxonômica e biogeográfica e padrões morfológicos dos cangambás (EIZIRIK et al., 2006; OLIVEIRA, 2006). Sabe-se que estes apresentam dieta oportunista, com riqueza de invertebrados (EMMONS; FEER, 1997; CHEIDA et al., 2011).

Estudos sobre a fauna parasitária baseiam-se na importância destes organismos para fornecer informações sobre a ecologia, o comportamento do hospedeiro e suas relações tróficas, assim como agentes causadores de doenças que podem influenciar na biosseguridade dos ecossistemas e dos ambientes naturais e domésticos (BROOKS; HOBERG, 2000).

No que tange à bioconservação, os parasitos podem atuar no controle populacional dos hospedeiros, auxiliar na manutenção do equilíbrio populacional, na variabilidade genética e na estruturação das comunidades de vertebrados e invertebrados (WINDSOR, 1995; 1997). Por outro lado, os organismos patogênicos podem representar uma ameaça para os programas de manejo e recuperação de espécies ameaçadas (HOLMES, 1996). Alguns autores têm ressaltado o papel das doenças infecciosas na perda da biodiversidade, devido à mortalidade em massa e declínio populacional (HANSELMANN et al., 2004; DASZAK et al., 2005; SCHLOEGEL et al., 2006). Algumas populações densas em ambientes fragmentados podem enfrentar pressão direta e crescente ocasionada por doenças parasitárias como consequência de diversos fatores: (1) estresse social ocasionado pelo aumento da densidade populacional, (2) efeitos deletérios da consanguinidade, (3) deterioração da qualidade do hábitat, (4) baixa disponibilidade de alimento entre outros. Estes fatores associados ao estado nutricional do animal, suas condições fisiológicas, idade e carga parasitária podem ser responsáveis por uma maior morbidade (PRIMACK; RODRIGUES, 2001; BUSH et al., 2001).

O presente trabalho teve por objetivo relatar a ocorrência de parasitos em animais da espécie $C$. semistriatus do estado de Minas Gerais. Para tal, foi realizado um estudo transversal com uma amostra de conveniência constituída de dois animais resgatados e transportados para o Laboratório de Anatomia Animal da Universidade Federal de Lavras, MG. A coleta dos cadáveres foi realizada com autorização do ICMBIO № 31887-1.

Os animais foram identificados como $C$. semistriatus (jaritataca), inspecionados para verificação da presença de ectoparasitos e em seguida dissecados para recuperação do conteúdo gastrintestinal e pesquisa de helmintos.

Não foram identificados ectoparasitos, mas foi identificada a presença de seis helmintos aderidos à mucosa do estômago de um dos animais, sendo três machos e três fêmeas. Os machos mediam 21$30 \mathrm{~mm}$ de comprimento e 0,91-1,32 $\mathrm{mm}$ de largura. As fêmeas eram um pouco maiores, medindo de 28 $47 \mathrm{~mm}$ de comprimento por 1,2-1,6 $\mathrm{mm}$ de largura. 
Os processamento dos espécies foi realizado conforme descrito por (MOLINARO et al., 2012). Os espécimes foram fixados em AFA (93 partes de etanol 70\%; 5 partes de formalina e 2 partes de ácido acético glacial) por um período de $48 \mathrm{~h}$. Após fixação, os parasitos foram acondicionados em etanol $70 \%$ contendo $5 \%$ de glicerina. Para o propósito de visualização das estruturas internas e identificação morfológica e morfométrica, os parasitos foram clarificados em Lactofenol de Amann (1:1:2:1 fenol:ácido lático: glicerina: água destilada).

A mensuração dos parasitos foi realizada com a utilização de ocular micrométrica, em microscópio Olympus XC21. A identificação dos espécimes até gênero foi feita utilizando-se os trabalhos de Yamaguti (1961), Travassos et al. (1969) e Chabaud (1974). Os exemplares examinados foram identificados como pertencentes ao gênero Physaloptera (Spirurida: Physalopteridae), pela presença de colarete cefálico, boca com pseudolábios proeminntes e providos de estruturas semelhantes a dentículos.

Ao exame macroscópico verificou-se a presença nodulações na mucosa estomacal associadas ao parasitismo desenvolvido por estes nematoides. Fragmentos deste tecido hospedeiro foram coletados e fixados em solução de formalina tamponada $10 \%$ ( $\mathrm{pH} 7,2$ e 0,01 M) para análise histopatológica. Após fixação, o material foi emblocado em parafina, e cortados em seções de $5 \mu \mathrm{m}$. O material foi corado com azul de Toluidina, Grocott e contracorado com hematoxilinaeosina. Após coloração, o material foi examinado usando o microscópio de luz Olympus XC21 (NAEM et al., 2006).

Ao exame microscópico verificou-se que na submucosa haviam granulomas coalescentes, caracterizados por áreas centrais de necrose, com restos de parasitos margeados por células gigantes de Langhans, acentuado infiltrado de eosinófilos e menor número de células mononucleares e eosinófilos.

À análise histopatológica, verificou-se ainda proliferação de tecido conjuntivo fibroso circunjacente e infiltrado de eosinófilos na submucosa e lâmina própria adjacente aos granulomas. Ausência de mastócitos no Azul de Toluidina. Grocott negativo para fungo. A lesão observada foi classificada como de origem parasitária, representando o ponto de fixação dos parasitos do gênero Physaloptera observáveis no lúmen do estômago.

A pesquisa de parasitos em animais silvestres é uma ferramenta importante no estudo do estado sanitário de uma população e da qualidade do ambiente, podendo ser utilizada em ações de manejo da fauna (CATENACCI et al., 2004). A ocorrência de parasitos do gênero Pshysaloptera já foi relatada em seis famílias de carnívoros: Felidae, Canidae, Procyonidae, Mustelidae, Hyaenidae e Veverridae (MORGAN, 1944). Embora as infecções helmínticas, geralmente, não sejam responsáveis por uma alta letalidade, estas contribuem para um mau estado de saúde, afetando inclusive o comportamento dos animais parasitados, que ficam mais suscetíveis à predação ou a outras pressões ambientais (SANTOS, 2008). Assim, infecção parasitária pode passar despercebida ou causar aumento da taxa de mortalidade ou incremento da morbidade. Considerando serem raros, no Brasil, estudos que associam o parasitismo, ecologia do hospedeiro, degradação e/ou recuperação ambiental, trabalhos relacionados à fauna parasitária da espécie $C$. semistriatus torna-se um dado relevante. A realização de mais estudos sobre parasitos deste vertebrado podem fornecer informações adicionais sobre a patogenia das infecções, contribuindo para a identificação de animais parasitados.

\section{Agradecimentos}

Os autores agradecem à Fundação de Amparo a Pesquisa do Estado de Minas Gerais (FAPEMIG), ao BIOPAR, à CAPES e ao CNPq pelo apoio concedido.

\section{Referências}

BROOKS, D. R.; HOBERG, E. P. Triage for the biosphere: the need and rationale for taxonomic inventories and phylogenetic studies of parasites. Comparative Parasitology, Lawrence, v. 67, n. 1, p. 1-25, 2000.

BUSH, A. O.; FERNÁNDEZ, J. C.; ESCH, G. W.; SEED, J. R. Parasitism: the diversity and ecology of animal parasites. Cambridge: Cambridge University Press, 2001. 566 p.

CATENACCI, L. S.; VELASTIN, G. O.; ROCHA, F. S. A fragmentação de habitat aumenta a intensidade de enteroparasitas em gambás de orelhas brancas (Didelphis albiventris). In: 
CONGRESSO E ENCONTRO DA ASSOCIAÇÃO BRASILEIRA DE VETERINÁRIOS DE ANIMAIS SILVESTRES, VIII, XIII, 2004, Jaboticabal. Resumos... Jaboticabal: ABRAVAS, 2004. Versão eletrônica.

CHABAUD, A. G. Class Nematoda: keys of the subclasses, orders, and superfamilies. In: CHABAUD, A. G.; ANDERSON, R.C.; WILLMONT, S. (Ed.). Keys to the nematode parasites of vertebrates. 1. ed. London: Farnham Royal, 1974. p. 6-17.

CHEIDA, C. C.; NAKANO-OLIVEIRA, E.; FUSCO-COSTA, R.; ROCHAMENDES, F.; QUADROS, J. Ordem Carnivora. In: REIS, N. R.; PERACCHI, A. L.; PEDRO, W. A.; LIMA, I. P. (Ed.). Mamíferos do Brasil. 2. ed. Londrina: Imprensa da UEL, 2011. p. 235-288.

DASZAK, P.; SCOTT, D. E.; KILPATRICK, A. M.; FAGGIONI, C.; GIBBONS, J. W.; PORTER, D. Amphibian population declines at savannah river site are linked to climate, not chytridiomycosis. Ecology, New York, v. 86, p. 3232-3237, 2005.

DRAGOO, J. W.; HONEYCUTT, R. L. Systematics of mustelid like carnivores. Journal of Mammalogy, Lawrence,v. 78, p. 426443, 1997.

DRAGOO, J. W.; HONEYCUTT, R. L.; SCHIMDLY, D. J. Taxonomic status of white-backed hog-nosed skunks, genus Conepatus (Carnivora: Mephitidae). Journal of Mammalogy, Lawrence, v. 84, p. 159-176, 2003.

EIZIRIK, E.; JOHNSON, W. E.; O'BRIEN, S. J. Definindo unidades evolutivamente significativas e unidades de manejo para a conservação de carnívoros neotropicais. In: MORATO, R. G.; RODRIGUES, F. H. G.; EIZIRIK, E.; MANGINI, P. R.; AZEVEDO F. C. C.; MARINHO-FILHO, J. S. (Ed.). Manejo e conservação de carnívoros neotropicais. São Paulo: IBAMA, 2006. p. 47-66.

EMMONS, L.; FEER, F. Neotropical rainforest mammals: a field guide. Chicago: University of Chicago, 1997. 396 p.

HANSELMANN, R.; RODRIGUEZ, A.; LAMPO, M.; RAMOS, L. F.; AGUIRRE, A. A.; KILPATRICK, A. M.; RODRIGUEZ, J. P.; DASZAK, P. Presence of an emerging pathogen of amphibians in introduced bullfrogs Rana catesbeiana in Venezuela. Biological Conservation, Boston, v. 120, p. 115-119, 2004.

HOLMES, J. C. Parasites as threats to biodiversity in shrinking ecosystems. Biodiversity and Conservation, New York, v. 5, p. 975-983, 1996.
MOLINARO, E. M.; CAPUTO, L. F. G.; AMENDOEIRA, M. R. $R$. Conceitos e métodos para a formação de profissionais em laboratórios de saúde. Rio de Janeiro: Escola Politécnica de Saúde Joaquim Venâncio, 2012. 476 p.

MORGAN, B. B. The Physaloptera Nematoda of carnivores. Transactions of the Wisconsin Academy of Sciences Arts and Letters, Wisconsin, v. 36, p. 375-388, 1944.

NAEM, S.; FARSHID, A. A.; MARAND, M. T. Pathologic findings on natural infection with Phyasaloptera praeputialis in cats. Veterinarski Arhiv, Zagreb, v. 76, p. 315-321, 2006.

OLIVEIRA, T. G. Research in terrestrial carnivora from Brazil: current knowledge and priorities for the new millenium. In: MORATO, R. G.; RODRIGUES, F. H. G.; EIZIRIK, E.; MANGINI, P. R.; AZEVEDO F. C. C.; MARINHO-FILHO, J. S. (Ed.). Manejo e conservação de carnívoros neotropicais. São Paulo: IBAMA, 2006. p. 47-66.

PRIMACK, R. B.; RODRIGUES, E. 2001. Biologia da Conservação. Londrina: Editora Vida, 2001. 327 p.

SANTOS, J. L. C. Parasitos de canídeos domésticos e silvestres da região do Parque Nacional da Serra do Cipó - Minas Gerais - Brasil. 2008. 120 f. Dissertação (Mestrado em Parasitologia) Universidade Federal de Minas Gerais. Belo Horizonte, 2008.

SCHLOEGEL, L. M.; HERO, J.; BERGER, L.; SPEARE, R.; MCDONALD, K.; DASZAK, P. The decline of the sharp-snouted day frog (Taudactylus acutirostris): the first documented case of extinction by infection in a free-ranging wildlife species? EcoHealth, New York, v. 3, p. 35-40, 2006.

TRAVASSOS, L.; FREITAS, J. F. T.; KONH, A. Trematódeos do Brasil. Memórias do Instituto Oswaldo Cruz, Rio de Janeiro, v. 67, p. 11-865, 1969.

WINDSOR, D. A. Equal rights for parasites. Conservation Biology, San Francisco, v. 9, n. 1, p. 1-2, 1995.

WINDSOR, D. A. Stand up for parasites. Trends in Ecology and Evolution, Cambridge, v. 12, p. 32, 1997.

YAMAGUTI, S. Systema Helminthum. New York: Interscience Publisher, 1961. $679 \mathrm{p}$. 